\title{
ARTICLE Identification of PRDX6 as a regulator of ferroptosis
}

\author{
Bin $\mathrm{Lu}^{1}$, Xiao-bing Chen ${ }^{1}$, Yu-cai Hong ${ }^{2}$, Hong Zhu ${ }^{1}$, Qiao-jun $\mathrm{He}^{1}$, Bo Yang ${ }^{1}$, Mei-dan Ying ${ }^{1}$ and Ji Cao ${ }^{1}$
}

Ferroptosis is a newly characterized iron-dependent form of nonapoptotic regulated cell death triggered by lipid reactive oxygen species (LOOH). The dysregulation of ferroptosis is highly related to cancer, and the induction of ferroptosis is also proposed as a potential strategy for cancer therapy. Although several key regulators have been identified that are involved in ferroptosis, the molecular mechanism underlying this process remains largely unknown. Here, we report that Peroxiredoxin-6 (PRDX6) is a bona fide negative regulator of ferroptotic cell death. The knockdown of intracellular PRDX6 significantly enhances LOOH and ferroptotic cell death triggered by ferroptosis inducers (Erastin and RSL-3), which is correlated with the transcriptional activation of heme oxygenase-1. Moreover, overexpression of heme oxygenase-1 enhances both Erastin- and RSL-3-triggered LOOH, suggesting that heme oxygenase-1 mediates PRDX6 silencing-enhanced ferroptosis. More importantly, the application of a specific PRDX6 phospholipase A2 (iPLA2) inhibitor, MJ-33, synergistically enhances the ferroptosis induced by Erastin, suggesting that PRDX6 removes $\mathrm{LOOH}$ through its iPLA2 activity. Thus, our findings reveal an essential role of PRDX6 in protecting cells against ferroptosis and provide a potential target to improve the antitumor activity of ferroptosis-based chemotherapy.

Keywords: PRDX6; ferroptosis; iPLA2 activity; heme oxygenase-1; antitumor therapy

Acta Pharmacologica Sinica (2019) 40:1334-1342; https://doi.org/10.1038/s41401-019-0233-9

\section{INTRODUCTION}

Ferroptosis is a newly characterized nonapoptotic form of programmed cell death dictated by cell membrane oxidative damage [1-4]. The overwhelming accumulation of phospholipid (PL) hydroperoxides (such as lipid ROS or lipid reactive oxygen species (LOOH) is the hallmark [1] and the known cause of ferroptosis, which originates from the peroxidized cell membrane. Increasing evidence suggests that dysfunctional ferroptosis is related to several severe human diseases, including cancer [5-8]. It was reported that $\mathrm{p} 53$ carries out its tumor-suppressive functions partially by increasing the sensitivity of the tumor cell to ferroptosis [9, 10]. In addition, ferroptotic inducers (such as $(1 S, 3 R)-R S L 3)$ exhibit a significant preventive and therapeutic effect against tumors in a xenograft mouse model [11]. More excitingly, ferroptotic inducers can overcome resistance to chemotherapy drugs (such as cisplatin [12], cytarabine and doxorubicin [13]) and enhance their effects on certain tumor cells. Therefore, it has recently been recognized that ferroptosis plays an important role in tumorigenesis, and thus, the activation of ferroptosis holds great potential for tumor therapeutics.

Given that increasing evidence suggests the critical roles of ferroptosis in cancer, it is of particular importance to understand its regulatory mechanism, which remains largely unknown at present. The selenoprotein glutathione peroxidase 4 (GPX4), a key enzyme for repairing peroxidized cell membranes, is the bestdefined protein regulator of ferroptosis [11, 14]. When GPX4 activity is inhibited, ferroptosis will be initialized, resulting in ferroptotic process. In addition, the cell-surface $\mathrm{Na}^{+}$-independent glutamate-cystine antiporter system $x_{c}^{-}[15]$ is another important protein that serves as an upstream regulator of GPX4 by determining the intracellular concentration of glutathione (GSH), which is an essential cofactor of GPX4. Moreover, several negative regulators of ferroptosis have been reported over the past few years. For example, heat shock protein beta-1 (HSPB1) [16] and nuclear factor erythroid 2-related factor 2 (NRF2) [17]-mediated iron and ROS metabolism are critically involved in protecting tumor cells against ferroptosis. In addition, two enzymes involved in fatty acid metabolism, ACSL4 and LPCAT3, preferentially catalyzing PLs containing unsaturated fatty acids (PUFA) esterification into PLs and providing available PUFA-PLs for ferroptosis, are reported as key positive ferroptotic regulators $[3,18]$. Although the main pathway of ferroptosis has been established, the exact regulatory network is still largely unknown.

Peroxiredoxins (PRDXs) are an important family of nonselenium peroxidases, consisting of six members $[19,20]$. According to the number of conserved cysteine residues in the catalytic center, these proteins can be classified into two groups, PRDX1-5 (containing two cysteines) and PRDX6 (containing one cysteine). In addition to this difference, PRDX1-5 use thioredoxin (Trx) as the physiological reductant to conduct peroxidase activities, while PRDX6 uses GSH, which is the same as GPX4. In addition, several studies have demonstrated that PRDX6 plays a major role in the repair of peroxidized cell membranes by two mechanisms [21, 22]. On the one hand, PL hydroperoxides are reduced to alcohols through the PL hydroperoxidase activity of PRDX6 and are further reduced by a series of nonspecific dehydrogenases to complete regeneration. On the other hand, PL hydroperoxides are hydrolyzed at their sn-2 fatty acyl ester bond to peroxidized PUFA and lysophospholipid (LPC) through $\mathrm{Ca}^{2+}$-independent phospholipase A2 activity of PRDX6; afterward, LPC is reacetylated with free

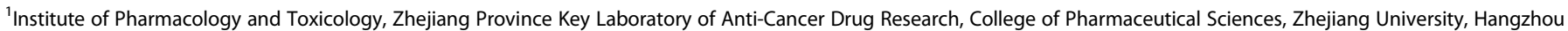
310058, China and 2Department of Emergency Medicine, Sir Run Run Shaw Hospital, Zhejiang University School of Medicine, Hangzhou 310016, China Correspondence: Ji Cao (caoji88@zju.edu.cn) 
FUFA-CoA through the LPC acyl transferase activity of PRDX6 [23] to complete regeneration. Given the significant similarity between PRDX6 and GPX4 regarding their enzymatic activity and the core position of GPX4 in ferroptosis, we are greatly interested in exploring the role of PRDX6 in ferroptosis. Thus, this study investigated the role of PRDX6 in modulating ferroptosis. The data presented in this work demonstrate a novel role for PRDX6 in the regulation of ferroptosis and provide new insight into how cancer cells defend themselves against ferroptotic processes.

\section{MATERIALS AND METHODS}

Antibodies and reagents

The primary antibody against PRDX6 (\#13585, 1:1000) was purchased from Proteintech (USA). The antibody against NRF2 (sc13032, 1:500) was purchased from Santa Cruz Biotechnology (USA). The antibody against Keap1 (P586, 1:1000) was purchased from Cell Signaling Technology (USA). The antibodies against Actin (db1959, 1:2000) and FLAG (db7002, 1:1000) were purchased from Diagbio (China). Erastin (S7242), ferrostatin-1 (S7243), and RSL-3 (S8155) were purchased from Selleck (USA) and dissolved in DMSO. Sorafenib (S125098) was purchased from Aladdin (China) and dissolved in DMSO. MJ33 (C5346) was purchased from APEXBIO (USA) and dissolved in $\mathrm{H}_{2} \mathrm{O}$ (warmed to $60^{\circ} \mathrm{C}$ ). Doxycycline hyclate (D9891) and deferoxamine (D9533) were purchased from Sigma-Aldrich (USA) and dissolved in $\mathrm{H}_{2} \mathrm{O}$ and DMSO, respectively. Necrostatin-1 (MB5067) was purchased from Meilunbio (China) and dissolved in DMSO. Z-VAD-FMK (C1202) was purchased from Beyotime (China) and dissolved in DMSO. Geneticin (\#11811031) was purchased from Thermo Fisher Scientific (USA) and dissolved in $\mathrm{H}_{2} \mathrm{O}$.

The full-length coding sequences of PRDX6 and HMOX 1 were amplified from the H1299 and SW480 cDNA libraries, respectively, and subsequently subcloned into the $\mathrm{pCDH}$ plasmid.

\section{Cell culture}

The H1299, A549, and 293FT cell lines were purchased from the Shanghai Institute of Biochemistry and Cell Biology (Shanghai, China). Following receipt, the cells were grown and frozen as seedstock as soon as they were available. All cell lines were passaged for a maximum of 2 months, after which new seedstocks were thawed. All cell lines used were authenticated using shorttandem repeat profiling every 6 months. All cell lines were tested for mycoplasma contamination at least once per month. H1299 and A549 cells were cultured in RPMI-1640 medium (SigmaAldrich, USA). 293FT cells were cultured in Dulbecco's modified Eagle's medium (DMEM) medium (Sigma-Aldrich, USA). All of the media were supplemented with $10 \%$ fetal bovine serum (HyClone, SV30160.03, GE Healthcare, USA), 100 units $/ \mathrm{mL}$ penicillin and 100 $\mu \mathrm{g} / \mathrm{mL}$ streptomycin. All of the cells were incubated at $37^{\circ} \mathrm{C}$ in a humidified atmosphere with $5 \% \mathrm{CO}_{2}$.

Lentivirus production and transduction

The lentiviral shRNA vector pLKO.1-puro (SHC001) was obtained from Sigma-Aldrich (USA). Human PRDX6-shRNA1 (NM_004905.2-271s1c1; sequence: 5'-CCGGGACAGTGTTGAGGAC CATCTTCTCGAGAAGATGGTCCTCAACACTGTCTITTG-3') and human PRDX6-shRNA2 (NM_004905.2-658s1c1; sequence: 5'CCGGCCGAAAGGAGTCTTCACCAAACTCGAGTITGGTGAAGACTCCT TTCGGTITTG-3') were obtained from Sigma-Aldrich (USA). Recombinant lentiviruses were produced by cotransfecting 293FT cells with lentiviral PRDX6 shRNA expression plasmids, pCMV-R8.91 plasmids (packaging vector) and pMD2-VSVG plasmids (envelope vector), at a ratio of 5:5:1, using PEI MAX 40K (\#24765, Polysciences, USA) as a transfection reagent. At $16 \mathrm{~h}$ after transfection, the medium was removed and subsequently replaced with culture medium containing $1 \mathrm{mM}$ sodium pyruvate. Virus-containing medium was harvested $32 \mathrm{~h}$ later, centrifuged and filtered with a $0.45 \mu \mathrm{m}$ filter. The day before the transduction, 100,000 cells per well were seeded in a 6-well plate. The day of the transduction, cells were transduced with $0.5 \mathrm{~mL}$ of lentiviruscontaining medium supplemented with $2.5 \mu \mathrm{L}$ polybrene $(6 \mathrm{mg} /$ $\mathrm{mL}$ ) for $12 \mathrm{~h}$. The medium was then removed and replaced with fresh culture medium. The lentiviral shRNA vector pLKO.1-puro was used as a control.

Plasmid transfection

The day before the transfection, 250000 cells (for H1299) were seeded on six-well plates. The day of the transfection, cells were treated with jetPRIME ${ }^{\infty}$ according to the manufacturer's instructions.

Tetracycline-inducible lentiviral knockdown

The tetracycline-inducible lentiviral knockdown plasmid (TetpLKO-neo) was kindly provided by Dr. He-ning Lin, Cornell University. The human PRDX6-shRNA was consistent with human PRDX6-shRNA1. The lentivirus production and transduction were performed as mentioned above. Positive transductants were selected with geneticin $(800 \mu \mathrm{g} / \mathrm{mL})$.

Analysis of $\mathrm{LOOH}$ production

The day before the experiment, 120000 cells (for H1299) or 100 000 cells (for A549) per well were seeded in 6-well plates. The day of the experiment, cells were treated with different test compounds for the indicated times; harvested by trypsinization; resuspended in $500 \mu \mathrm{L} 1 \times$ phosphate-buffered solution (PBS) containing $2 \mu \mathrm{M}$ C11-BODIPY(581/591) (\#D3861, Invitrogen, USA) and incubated for $30 \mathrm{~min}$ at $37^{\circ} \mathrm{C}$ in a cell culture incubator. Cells were centrifuged at $1400 \mathrm{r} / \mathrm{min}$ at room temperature for $5 \mathrm{~min}$, the supernatants were removed, resuspended in $500 \mu \mathrm{L}$ of fresh $1 \times$ PBS, strained through a $40 \mu \mathrm{m}$ cell strainer, and analyzed using a flow cytometer (FACSuite, BD Biosciences, USA) equipped with a $488 \mathrm{~nm}$ laser for excitation. Data were collected from the FL1 channel $(527 \mathrm{nM})$. A minimum of 10000 cells were analyzed per condition.

\section{Cell survival assay}

Cell survival was assessed with a sulforhodamine B colorimetric assay (SRB, \#S1402, Sigma) [24]. Briefly, cell monolayers were fixed with $10 \%$ (wt/vol) trichloroacetic acid and stained for $30 \mathrm{~min}$, after which the excess dye was removed by washing repeatedly with $1 \%(\mathrm{vol} / \mathrm{vol})$ acetic acid. The protein-bound dye was dissolved in $10 \mathrm{mM}$ Tris-base solution for $O D$ determination at $515 \mathrm{~nm}$ using a microplate reader (Multiskan Spectrum, Thermo). Growth inhibition under test conditions was reported as a percentage relative to the negative control.

Intracellular GSH concentration determination

The relative GSH concentration in cell lysates was assessed using a kit (\#A006-2) from Nanjing Jiancheng Company (China) according to the manufacturer's instructions. The measurement of GSH used a kinetic assay in which catalytic amounts (nmol) of GSH caused a continuous reduction of 5,5'-dithiobis-(2-nitrobenzoic acid) to 5thio-2-nitrobenzoic acid and the GSSG formed was recycled by glutathione reductase and NADPH. The yellow product (5-thio-2nitrobenzoic acid) was measured spectrophotometrically at 420 $\mathrm{nm}$. The values were normalized to the protein concentrations and presented as percentages relative to the negative control.

Western blot analysis

Cells were lysed with $1 \%$ NP40 buffer $(50 \mathrm{mM}$ Tris- $\mathrm{HCl}, 150 \mathrm{mM}$ $\mathrm{NaCl}, 1 \% \mathrm{NP} 40, \mathrm{pH}=7.4,0.1 \mathrm{mM}$ sodium vanadate, $5 \mu \mathrm{g} / \mathrm{mL}$ leupeptin, $0.1 \mathrm{mM}$ phenyl methane sulfonyl fluoride) and incubated at $4^{\circ} \mathrm{C}$ for $30 \mathrm{~min}$. The lysate was then centrifuged at $14000 \times g$ at $4^{\circ} \mathrm{C}$ for $20 \mathrm{~min}$ to remove insoluble materials. The protein concentrations of whole-cell lysates were determined 
using the DC Protein Assay Kit (Bio-Rad, USA). Protein extracts were resolved by $8 \%-10 \%$ sodium dodecyl sulfate polyacrylamide gel electrophoresis and electroblotted onto polyvinylidene difluoride membranes (Millipore, USA). After being blocked with $5 \%$ nonfat milk, the membranes were incubated at $4{ }^{\circ} \mathrm{C}$ with various primary antibodies overnight and then visualized with appropriate HRP-conjugated secondary antibodies by enhanced chemiluminescence detection (NEL103E001EA, PerkinElmer) through AI600 (GE Healthcare, USA).

\section{Quantitative real-time polymerase chain reaction analysis}

The quantitative polymerase chain reaction (qPCR) analysis was performed as described previously [24]. Total RNA isolation was carried out with an RNAiso Plus kit (\#9109, TaKaRa, Japan) according to the manufacturer's instructions. Then, first-strand complementary DNA synthesis was performed using the Reverse Transcription System Kit (\#AT311-03, TransGen Biotech, China) according to the manufacturer's instructions. Quantitative real-time PCR (RT-PCR) analysis was performed using the $\mathrm{iTaq}^{\mathrm{TM}}$ Universal SYBR Green Supermix (\#172-5124, Bio-Rad, USA). The reaction mixtures containing SYBR Green were prepared following the manufacturer's protocol, and then $C T$ values were obtained using a qPCR platform (QuantStudio 6 Flex Real-Time PCR System, ThermoFisher Scientific, USA). The housekeeping gene actin was used as an internal standard. The primers used for the PCR were as follows:

ß-Actin: forward 5'-CACCATTGGCAATGAGCGGTTC-3', reverse 5'-AGGTCTITGCGGATGTCCACGT-3'; PRDX6: forward 5'-AGCTGTCTATCCTCTACCCAG-3', reverse 5'-GACCATCACACTATCCCCATC-3'; HMOX1: forward 5'-CCAGGCAGAGAATGCTGAGTTC-3', Reverse 5'-AAGACTGGGCTCTCCTTGTTGC-3'; NQO-1: forward 5'-GAAGAGCACTGATCGTACTGGC-3', reverse 5'-GGATACTGAAAGTTCGCAGGG-3';

FTH-1: forward 5'-TCCTACGTTACCTGTCCATGT-3', reverse 5'-GTTTGTGCAGTTCCAGTAGTGA-3';

FTL: forward 5'-TACGAGCGTCTCCTGAAGATGC-3', reverse 5'-GGTTCAGCTIITCTCCAGGGC-3'; GCLC: forward 5'-GAGGAACAATGTCCGAGTTC-3', reverse $5^{\prime}$-CTCCTCTCCTATGTCGGATA-3'; GCLM: forward 5'-TGAACTGGGGCCGCCTGCGG-3', reverse 5'-CAATGAACAGTTITGCAGAA-3'; NRF2: forward 5'-CAAAAGGAGCAAGAGAAAGCC-3', Reverse 5'-TCTGATTTGGGAATGTGGGC-3'.

Statistical analysis

All statistical analyses were performed using Prism 5.0c (GraphPad Software). The number of biological replicates for each experiment is indicated in the figure legends. Sample sizes were chosen based on standard experimental requirements in molecular biology. Differences between means were determined using two-tailed unpaired Student's $t$ tests and were considered significant at $P<0.05$.

\section{RESULTS}

PRDX6 is a bona fide negative regulator of ferroptotic cell death To determine the role of PRDX6 in ferroptosis, we generated an inducible knockdown $\mathrm{H} 1299$ cell line using the tetracyclineinducible lentiviral knockdown system. In this system, the expression level of intracellular PRDX6 is tightly controlled by doxycycline. As expected, the protein expression level of PRDX6 was dramatically decreased in PRDX6 shRNA cells, but not in control shRNA cells, after the cells were treated with doxycycline $(0.25 \mu \mathrm{g} / \mathrm{mL})$ for $48 \mathrm{~h}$ (Fig. 1a). Using these cells, we found that doxycycline-induced PRDX6 silencing did not result in upregulation of lipid ROS ( $\mathrm{LOOH})$, a well-characterized ferroptotic marker [1], as determined by flow cytometry using the fluorescent probe C11-BODIPY (Fig. 1b). Thus, under normal conditions, PRDX6 silencing may not be a factor that initiates the ferroptosis process.
Next, we asked whether the knockdown of PRDX6 could affect ferroptosis under ferroptotic stress. Therefore, we chose the specific ferroptosis inducer Erastin [1, 25], which has previously been shown to be able to dramatically increase the intracellular $\mathrm{LOOH}$ level in $\mathrm{H} 1299$ cells. We found that knockdown of PRDX6 after doxycycline treatment significantly increased Erastin-induced $\mathrm{LOOH}$ (from $24.33 \% \pm 2.76 \%$ to $49.95 \% \pm 2.70 \%$ ) in the genetically engineered $\mathrm{H} 1299$ cells (Fig. 1C). A similar result was also observed using another ferroptotic inducer, RSL-3 [11, 26] (Fig. 1d). To validate that PRDX6 knockdown specifically enhances the ferroptotic process, we used ferrostatin-1 [27], a specific inhibitor of ferroptosis. Notably, ferrostatin-1 (10 $\mu \mathrm{M})$ treatment completely reversed Erastin-induced $\mathrm{LOOH}$ accumulation in both control and PRDX6 knockdown cells (Fig. 1e). Thus, these findings indicate that under ferroptotic stress, knockdown of PRDX6 specifically and significantly amplifies ferroptosis.

To further confirm the role of PRDX6 in ferroptosis, we determined whether transient lentiviral knockdown of PRDX6 expression would also enhance the ferroptotic process. First, H1299 and A549 cells were infected with control and PRDX6 shRNA lentiviruses. PRDX6 expression was remarkably decreased after lentivirus infection (Fig. 2a and Fig. S1a). Next, these transient PRDX6 knockdown cells were treated with Erastin, and Erastin-induced $\mathrm{LOOH}$ was subsequently detected by flow cytometry. In line with data from the inducible lentiviral knockdown system, transient lentiviral knockdown of PRDX6 also significantly increased Erastin-induced $\mathrm{LOOH}$ both in $\mathrm{H} 1299$ and A549 cells (Fig. 2b and Fig. S1b, c). In H1299 cells, Erastininduced $\mathrm{LOOH}$ increased from $16.48 \% \pm 5.64 \%$ to $69.49 \% \pm$ $24.64 \%$ and $74.28 \% \pm 7.19 \%$ in two different shRNA sequences (Fig. 2b). Similar results were observed with two other ferroptotic inducers, RSL-3 and sorafenib [15], in H1299 cells (Fig. 2c).

Increased $\mathrm{LOOH}$ is a major cause of ferroptotic cell death. Indeed, we found that irregular cell morphology was gradually replaced by shrunken morphology in Erastin-treated H1299 cells (Fig. S1d). In line with the LOOH results, transient knockdown of PRDX6 significantly increased Erastin-induced cell death in H1299 cells (Fig. 2d and Fig. S1d). To validate whether PRDX6 knockdown specifically enhances the ferroptotic process, we used ferrostatin1, a specific inhibitor of ferroptosis [1,20]. Notably, Fer-1 treatment completely reversed Erastin-induced growth inhibition in both control and PRDX6 knockdown cells (Fig. 2d). Moreover, ferrostatin-1 but not necrostatin-1 (a necroptosis inhibitor, Nec1) or Z-VAD-FMK (an apoptosis inhibitor) inhibited $\mathrm{LOOH}$ in both control and PRDX6 knockdown cells (Fig. 2e and Fig. S1e). Thus, these findings indicate that under ferroptotic stress, transient lentiviral knockdown of PRDX6 also specifically and remarkably amplifies the ferroptosis-inducing effect.

Next, we asked whether ectopic expression of PRDX6 protein in H1299 cells could diminish Erastin-induced ferroptosis. As shown in Fig. 2f, g, overexpression of PRDX6 abolished Erastin-induced growth inhibition in $\mathrm{H} 1299$ cells. Furthermore, overexpression of PRDX6 inhibited both Erastin- and RSL-3-induced LOOH in H1299 cells (Fig. $2 \mathrm{~h}$, i). These data imply that PRDX6 overexpression contributes to ferroptosis resistance.

Taken together, the data above suggest that PRDX6 is a bona fide negative regulator of ferroptosis.

\section{PRDX6 can remove LOOH through its iPLA2 activity during} ferroptosis

To understand the mechanism by which PRDX6 negatively regulates ferroptosis, we first sought to determine whether PRDX6 knockdown could enhance the level of GSH depletion, which is required for this process. Blocking system $x_{\mathrm{c}}{ }^{-}$with Erastin or sorafenib inhibits cysteine-dependent GSH synthesis and subsequently results in ferroptosis $[1,15]$. Thus, GSH depletion is one of the most important molecular events to initialize ferroptotic cell death. However, PRDX6 knockdown could not further decrease 
a

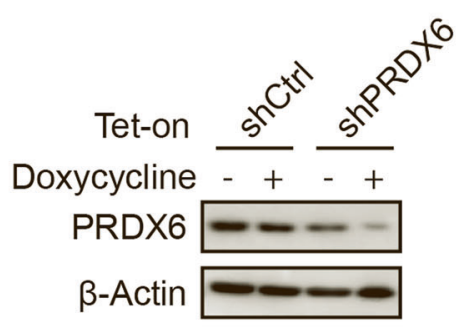

C

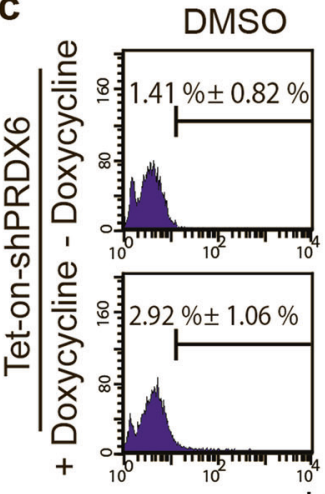

b Doxycycline
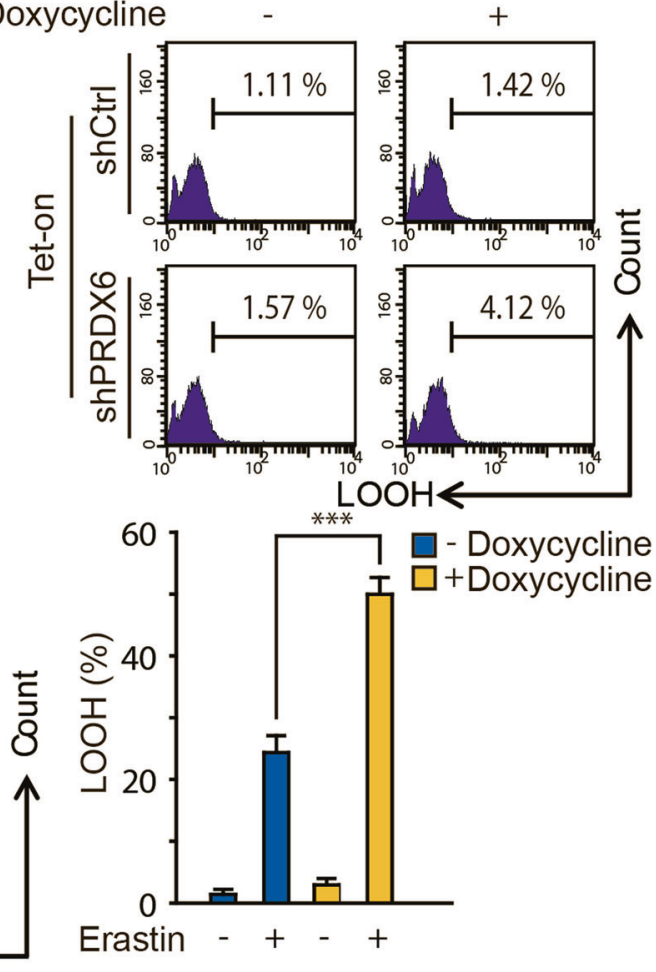

RSL-3

- Doxycycline

$\square+$ Doxycycline

d

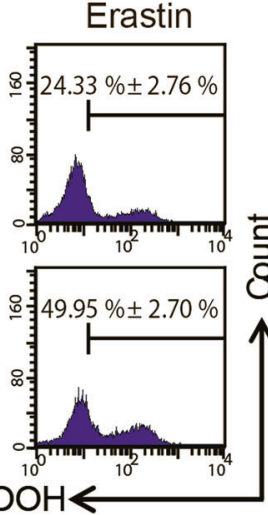

DMSO

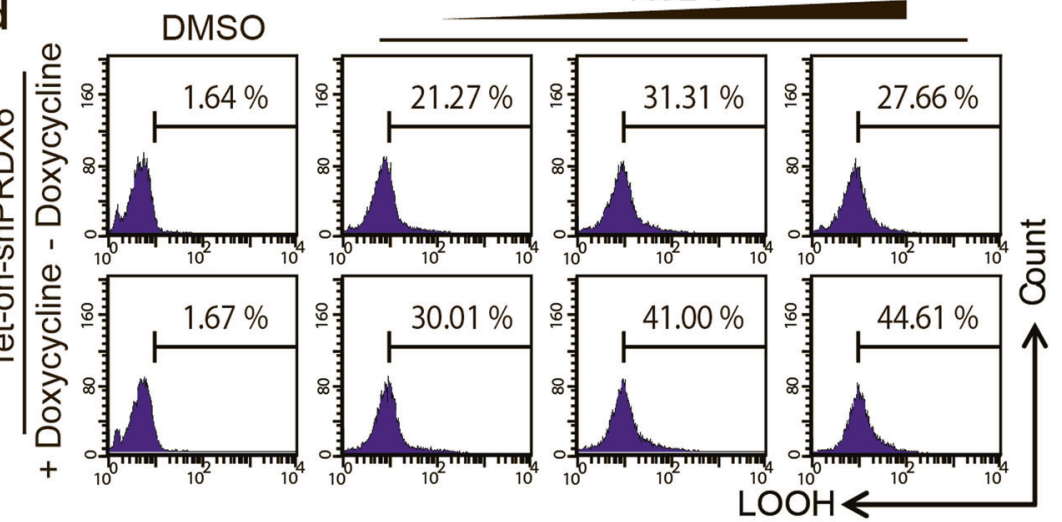

e
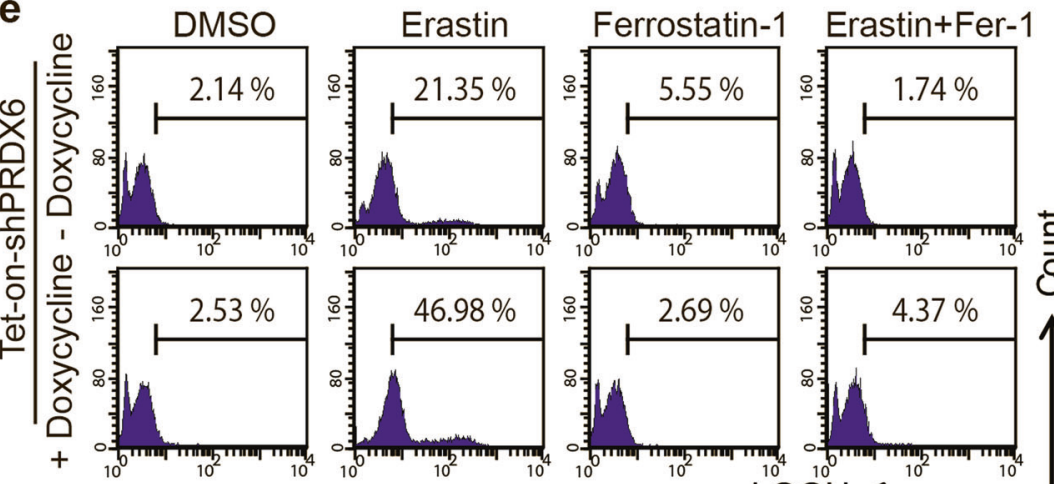

Fig. 1 PRDX6 knockdown through inducible lentiviral knockdown system enhances ferroptotic LOOH. a Western blot analysis of PRDX6 expression in indicated genetically engineered $\mathrm{H} 1299$ cells after doxycycline treatment $(0.25 \mu \mathrm{g} / \mathrm{mL})$ for $48 \mathrm{~h}, \mathbf{b}$ LOOH production was assayed by flow cytometry using C11-BODIPY. c After doxycycline $(0.25 \mu \mathrm{g} / \mathrm{mL})$ treatment for $48 \mathrm{~h}$, indicated genetically engineered $\mathrm{H} 1299 \mathrm{cells}$ were treated with Erastin $(2 \mu \mathrm{M})$ for $12 \mathrm{~h}$, and $\mathrm{LOOH}$ production was assayed. Representative data are shown and the statistical data are from three independent experiments and the bar indicates the mean value (***P<0.001). d After doxycycline treatment $(0.25 \mu \mathrm{g} / \mathrm{mL})$ for $48 \mathrm{~h}$, indicated genetically engineered $\mathrm{H} 1299$ cells were treated with RSL-3 (50, 100, or $150 \mathrm{nM})$ for $12 \mathrm{~h}$, and LOOH production was assayed. e After doxycycline $(0.25 \mu \mathrm{g} / \mathrm{mL})$ treatment for $48 \mathrm{~h}$, indicated genetically engineered $\mathrm{H} 1299$ cells were treated with Erastin ( $2 \mu \mathrm{M})$ with or without ferrostatin-1 $(10 \mu \mathrm{M})$ for $12 \mathrm{~h}$, and LOOH production was assayed 


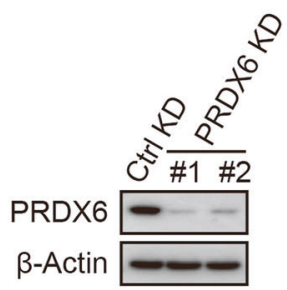

b

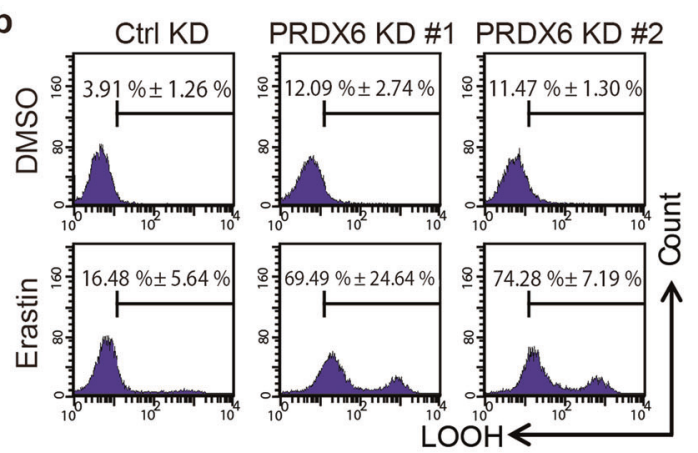

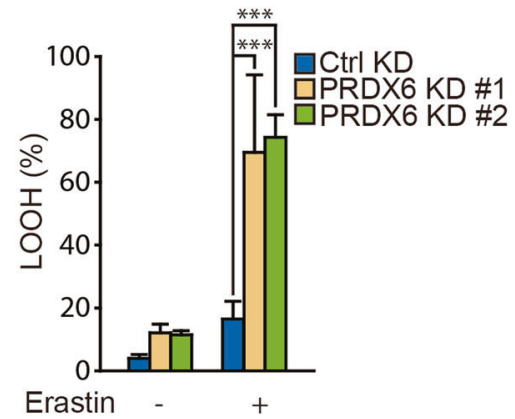

d
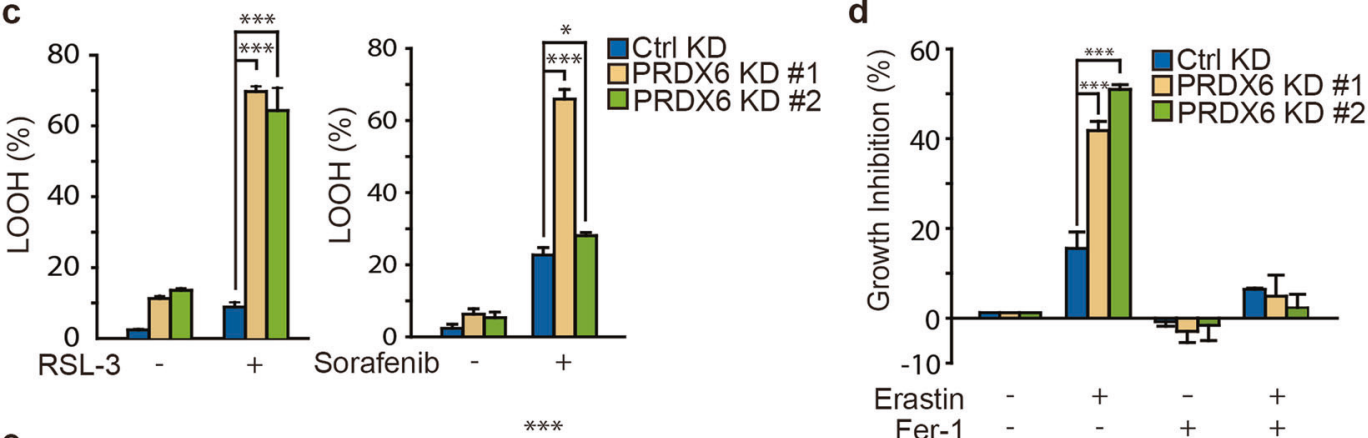

f
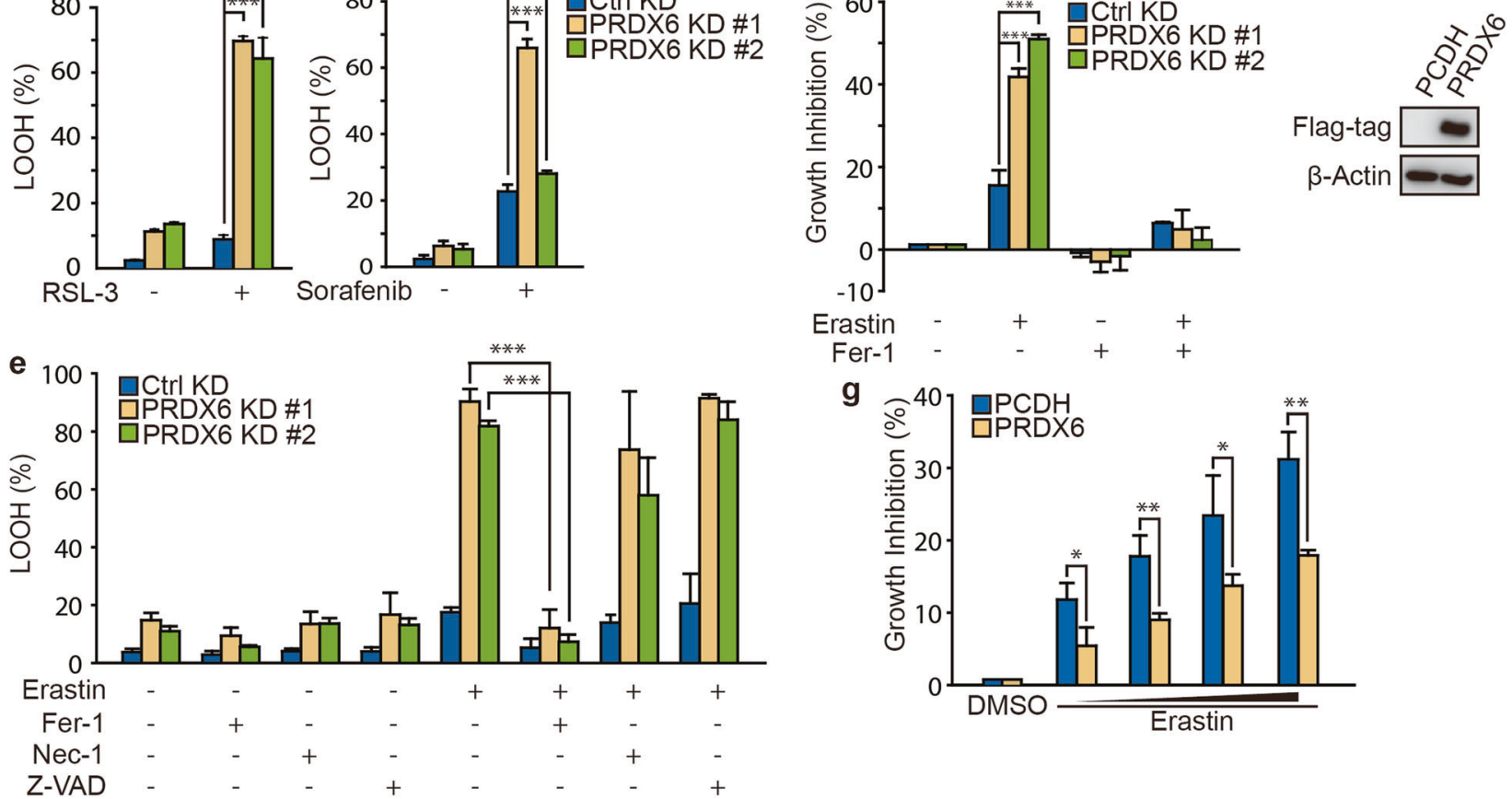

g

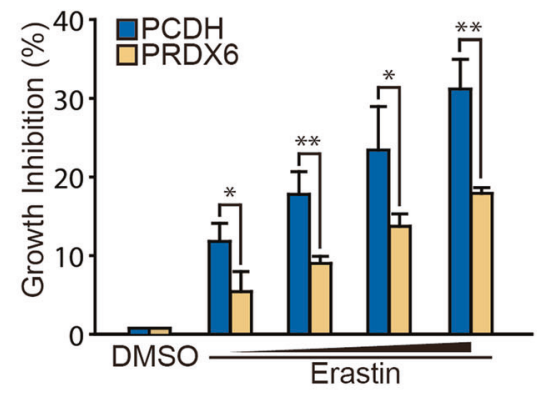

h
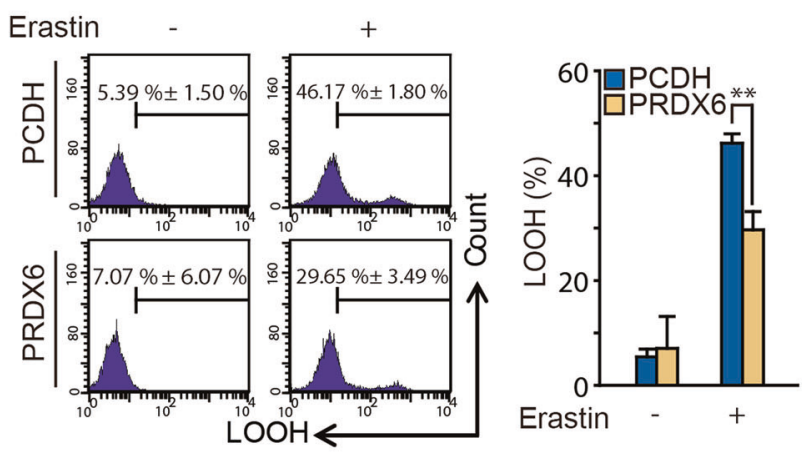

i
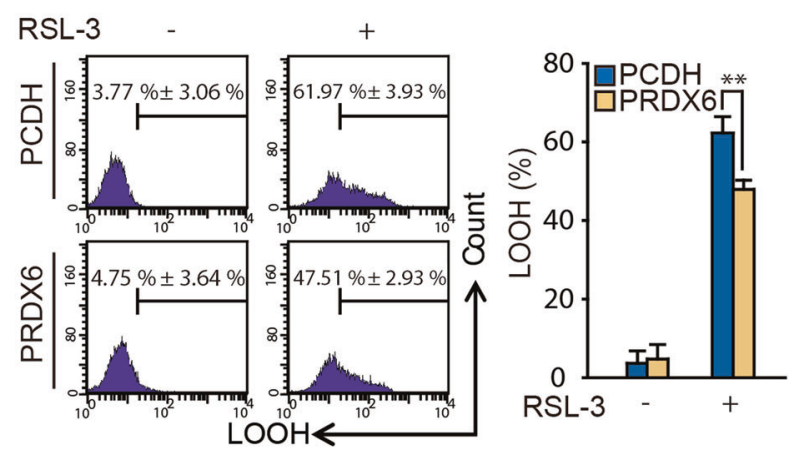

Fig. 2 PRDX6 knockdown through transient knockdown system enhances ferroptotic cell death. a Western blot analysis of PRDX6 expression in $\mathrm{H} 1299$ after infection of lentivirus targeting to PRDX6 for $48 \mathrm{~h}$. b After infection for $48 \mathrm{~h}, \mathrm{H} 1299$ cells was treated with Erastin $(2 \mu \mathrm{M})$ for $12 \mathrm{~h}$, and $\mathrm{LOOH}$ production was assayed by flow cytometry using C11-BODIPY. Representative data are shown and the statistical analysis is from nine independent experiments and the bar indicates the mean values. c After infection for $48 \mathrm{~h}, \mathrm{H} 1299$ cells were treated with RSL-3 (50 nM) or sorafenib $(20 \mu \mathrm{M})$ for $12 \mathrm{~h}$, and LOOH production was assayed by flow cytometry using C11-BODIPY. The statistical analysis is from three independent experiments and the bar indicates the mean values. d After infection for $48 \mathrm{~h}, \mathrm{H} 1299$ cells were treated for $24 \mathrm{~h}$ with Erastin $(2 \mu \mathrm{M})$ with or without ferrostatin-1(Fer-1) $(10 \mu \mathrm{M})$, and cell viability was assayed by SRB assay. The statistical analysis is from three independent experiments and the bar indicates the mean values. e After infection for $48 \mathrm{~h}, \mathrm{H} 1299$ cells were treated with Erastin ( $2 \mu \mathrm{M})$ with or without Fer-1 $(10 \mu \mathrm{M})$, necrostatin-1 $(30 \mu \mathrm{M})$ and Z-VAD-FMK $(10 \mu \mathrm{M})$ for $12 \mathrm{~h}$, and LOOH production was assayed. The statistical analysis is from three independent experiments and the bar indicates the mean values. $f$ Western blot analysis of PRDX6 overexpression in $\mathrm{H} 1299$ cells with transfection of PRDX6 cDNA. g After gene transfection for $24 \mathrm{~h}, \mathrm{H} 1299$ cells are treated with Erastin $(0,1,2,4$, and $8 \mu \mathrm{M})$ for $24 \mathrm{~h}$, and cell viability was assayed by SRB assay. The statistical analysis is from three independent experiments and the bar indicates the mean values. $\mathbf{h}, \mathbf{i}$ After gene transfection for $24 \mathrm{~h}, \mathrm{H} 1299$ cells are treated with Erastin $(8 \mu \mathrm{M})$ or RSL-3 $(100 \mathrm{nM})$ for $12 \mathrm{~h}$, and LOOH production was assayed. Representative data are shown and the statistical analysis is from three independent experiments and the bar indicates the mean values. $\left({ }^{*} P<0.05 ;{ }^{* *} P<0.01 ; * * * P<0.001\right)$ 


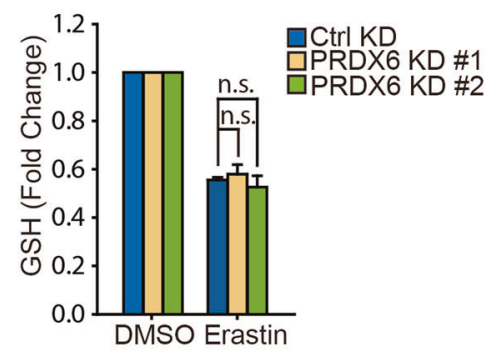

C

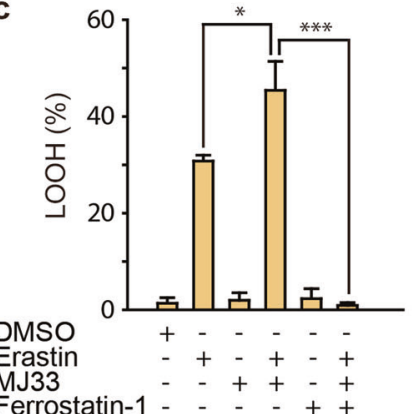

b
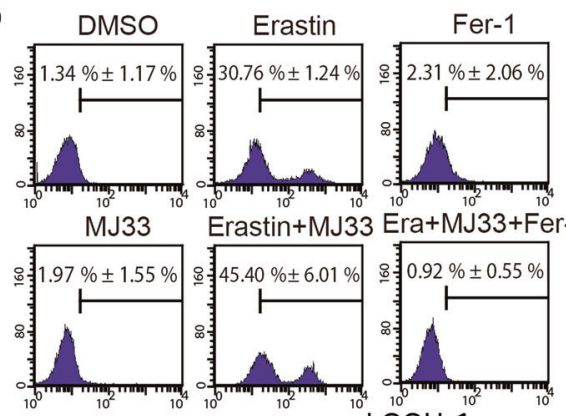

e

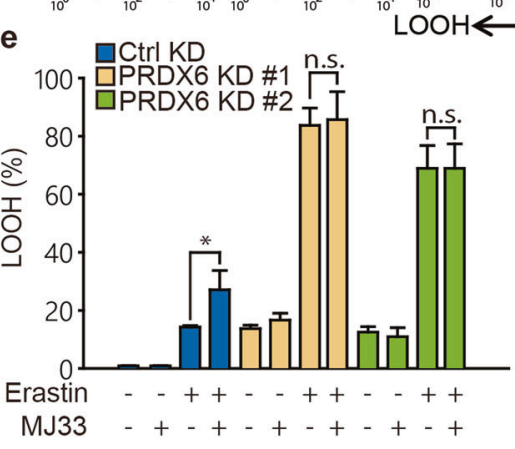

d
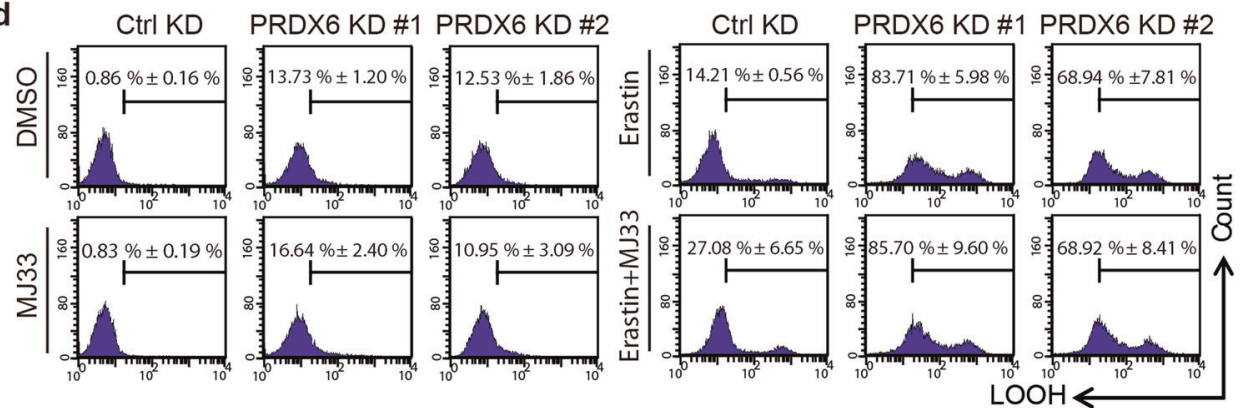

Fig. 3 PRDX6 negatively regulates ferroptosis through its iPLA2 activity. a After infection of lentivirus targeting to PRDX6 for $48 \mathrm{~h}, \mathrm{H} 1299$ cells were treated with Erastin $(2 \mu \mathrm{M})$ for $6 \mathrm{~h}$ and intracellular GSH levels were examined. The statistical analysis is from three independent experiments and the bar indicates the mean values. b, c H1299 cells were treated with Erastin $(2 \mu \mathrm{M})$ with or without of $\mathrm{MJ} 33(5 \mu \mathrm{M})$ or ferrostatin-1 $(10 \mu \mathrm{M})$ for $12 \mathrm{~h}$, and LOOH production was assayed by flow cytometry using C11-BODIPY. Representative data are shown and the statistical analysis is from three independent experiments and the bar indicates the mean values. d, e After infection for $48 \mathrm{~h}, \mathrm{H} 1299$ cells were treated with Erastin $(2 \mu \mathrm{M})$ with or without MJ33 $(5 \mu \mathrm{M})$ for $12 \mathrm{~h}$, and LOOH production was assayed. Representative data are shown and the statistical analysis is from three independent experiments and the bar indicates the mean values. $\left({ }^{*} P<0.05 ;{ }^{* *} P<0.001\right.$; n.s.: no statistic difference)

GSH levels compared to control knockdown after Erastin treatment in $\mathrm{H} 1299$ cells (Fig. 3a), suggesting that PRDX6 negatively regulating ferroptosis is independent of GSH depletion.

During the initial ferroptotic process, cell membrane PLs are peroxidized and subsequently result in PL hydroperoxide. Once GPX4 activity is inhibited or lost, accumulated PL hydroperoxide will result in ferroptosis. A recent study demonstrated that PRDX6 aids the repair of peroxidized cell membranes by PL hydroperoxide GPx (PHGPx) activity, $\mathrm{Ca}^{2+}$-independent phospholipase A2 (iPLA2) activity and LPC acyl transferase (LPCAT) activity [21]. Because there are no specific inhibitors of PHGPx of LPCAT, whereas a specific iPLA2 inhibitor (MJ33) is commercially available, we investigated the iPLA2 function of PRDX6 in ferroptosis. When the iPLA2 activity of PRDX6 was blocked using MJ33, there was no upregulation of $\mathrm{LOOH}$, suggesting iPLA2 activity of PRDX6 might not be an initial factor for inducing ferroptosis (Fig. 3b, c). Indeed, this result was consistent with the fact that PRDX6 silencing could also not upregulate $\mathrm{LOOH}$. However, under ferroptotic stress, MJ33 (5 $\mu \mathrm{M})$ significantly increased Erastin-induced $\mathrm{LOOH}$ in $\mathrm{H} 1299$ cells, from $30.76 \% \pm 1.24 \%$ to $45.40 \% \pm 6.01 \%$ (Fig. $3 \mathrm{~b}, \mathrm{c}$ ). In addition, increased $\mathrm{LOOH}$ could be completely reversed by ferrostatin-1. To rule out any potential off-target effects of MJ33, we further investigated the effect of MJ33 on PRDX6-silenced cells during Erastin treatment. As shown in Fig. 3d, e, MJ33 cannot further enhance the Erastin-induced $\mathrm{LOOH}$ accumulation in PRDX6-silenced cells, while MJ33 can still enhance Erastininduced $\mathrm{LOOH}$ accumulation in control cells. Furthermore, Erastin-induced $\mathrm{LOOH}$ accumulation gradually increased with increasing doses of MJ33 in A549 cells (Fig. S2a). These data suggest that PRDX6 removes a portion of LOOH through its iPLA2 activity during the ferroptotic process.

\section{PRDX6 silencing promotes the NRF2-mediated transcriptional activation of $H M O X 1$}

In addition to iPLA2 activity, we wonder whether there are other potential mechanisms that can explain how PRDX6 negatively regulates ferroptosis. Notably, under oxidative stress conditions, NRF2 activates the expression of the PRDX6 gene by a transcriptional mechanism involving an antioxidant response element [28]. In addition, a previous study demonstrated that NRF2 protects hepatocellular carcinoma cells against ferroptosis [17]. Therefore, we first investigated the role of NRF2 in PRDX6 silencing cells after Erastin treatment. Surprisingly, PRDX6 silencing significantly upregulated the expression of 


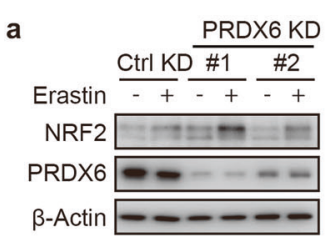

\section{b}

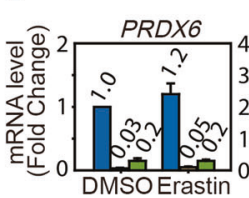

d
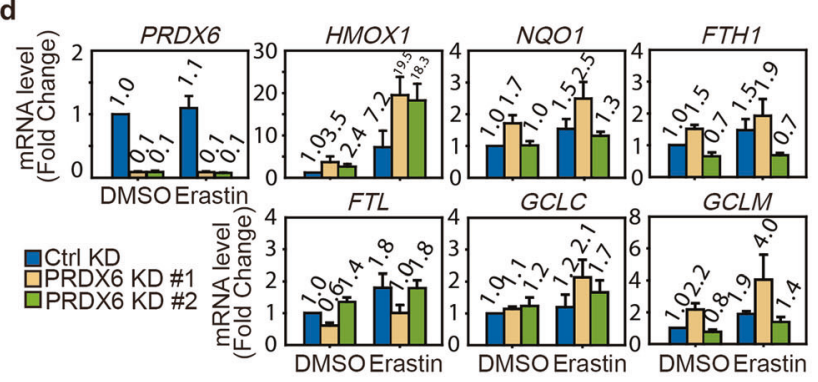

f
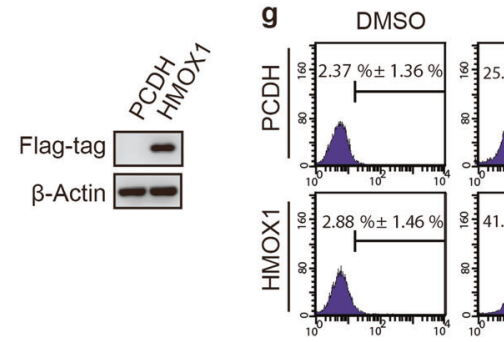

RSL-3

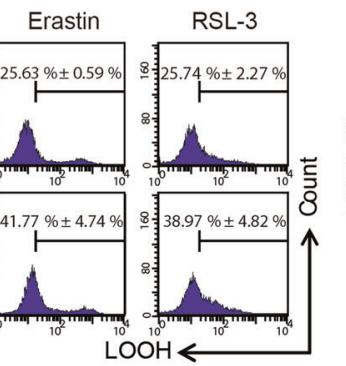

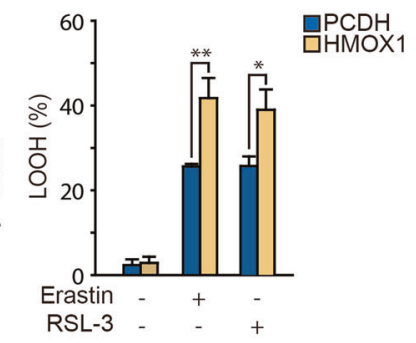

Fig. 4 PRDX6 silencing promotes the transcriptional activation of heme oxygenase-1. a, $\mathbf{b}$ After infection of lentivirus targeting to PRDX6 for $48 \mathrm{~h}, \mathrm{H} 1299$ cells were treated with Erastin $(2 \mu \mathrm{M})$ for $12 \mathrm{~h}$. a The protein expression of NRF2, and $\mathbf{b}$ its mRNA expression were assayed. The relative gene expression is normalized to $\beta$-actin and the error bar indicates the s.d. value from triplicates. c After infection for $48 \mathrm{~h}, \mathrm{H} 1299$ cells were treated with Erastin $(2 \mu \mathrm{M})$ for $12 \mathrm{~h}$, and Western blot analysis of Keap1 and PRDX6 expression. d After infection for $48 \mathrm{~h}, \mathrm{H} 1299$ cells were treated with Erastin $(2 \mu \mathrm{M})$ for $12 \mathrm{~h}$. These NRF2 downstream genes were assayed. The relative gene expression is normalized to $\beta$-actin and the error bar indicates the s.d. value from triplicates. e After infection for $48 \mathrm{~h}, \mathrm{H} 1299$ cells were treated with Erastin ( $2 \mu \mathrm{M})$ with or without DFO $(100 \mu \mathrm{M})$ for $12 \mathrm{~h}$, and $\mathrm{LOOH}$ production was assayed. Representative data are shown and the statistical analysis is from three independent experiments and the bar indicates the mean values. $\mathbf{f}$ Western blot analysis of HMOX 1 overexpression in H1299 cells with transfection of HMOX-1 CDNA. g After gene transfection for $24 \mathrm{~h}, \mathrm{H} 1299$ cells are treated with Erastin $(2 \mu \mathrm{M})$ or RSL-3 (50 nM) for $12 \mathrm{~h}$, and $\mathrm{LOOH}$ production was assayed. Representative data are shown and the statistical analysis is from three independent experiments and the bar indicates the mean values. $\left({ }^{*} P<0.05 ;{ }^{* *} P<0.01 ;{ }^{* * *} P<0.001\right)$

NRF2 after Erastin treatment in H1299 cells (Fig. 4a). To investigate how NRF2 protein is upregulated during ferroptosis in PRDX6silenced cells, we first analyzed the mRNA expression levels of NRF2 in PRDX6-silenced cells. However, NRF2 mRNA expression was not obviously changed in PRDX6-silenced cells treated with or without Erastin (Fig. 4b), indicating that upregulation of NRF2 expression occurs at the posttranscriptional stage. One possible mechanism is regulation of protein degradation. Keap1 is known to bind and retain NRF2 in the cytoplasm, where it can be targeted for ubiquitin-mediated degradation [29]; therefore, we decided to further investigate the role of Keap1 in PRDX6-silenced cells. As shown in Fig. 4c, the Keap1 protein level significantly decreased when PRDX6 was silenced in both the control and Erastin groups, suggesting that the upregulation of NRF2 is attributed to the decrease in Keap1 in PRDX6-silenced cells under ferroptotic stress. Given that ferroptosis is an iron-dependent and lipid ROSdependent form of cell death, we focused on analyzing the mRNA expression of the prototypical NRF2 target gene quinone oxidoreductase 1 (NQO1) and other target genes involved in heme and iron metabolism and GSH synthesis, including heme oxygenase 1 (HMOX1), ferritin heavy chain 1 (FTH1), ferritin light chain $(F T L)$, glutamate-cysteine ligase modifier subunit (GCLM), and glutamate-cysteine ligase catalytic subunit (GCLC). Among the above genes, the expression of HMOX1 was significantly upregulated in the PRDX6 knockdown cells compared with control cells after Erastin treatment (Fig. 4d). However, the expression of the other five genes did not change notably between the control and PRDX6 knockdown cells. A similar result was also observed in A549 cells (Fig. S3a). A previous study demonstrated that HMOX1 accelerates Erastin-induced ferroptosis through enzymatic degradation products of heme, including hemin and CO-releasing molecules [30]. In addition, HMOX1 has been shown to regulate cellular iron homeostasis of both free and chelatable iron, which contributes to the accumulation of ferrous ions [31]. Treatment with deferoxamine (DFO), an iron chelator, significantly ameliorated Erastin-induced $\mathrm{LOOH}$ in both the control and PRDX6silenced cells (Fig. 4e and Fig. S3b). Furthermore, overexpression of HMOX1 by transfection of HMOX1-complementary DNA (cDNA) increased both Erastin- and RSL-3-induced LOOH in H1299 cells (Fig. 4f, g). These results indicated that HMOX1 enhances the ferroptotic process in PRDX6-silenced cells by promoting cellular accumulation of ferrous ions. Collectively, these results indicated that after Erastin treatment, PRDX6 knockdown resulted in NRF2 activation, activation of HMOX1 transcription, and, eventually, an enhanced ferroptotic process.

\section{DISCUSSION}

Since the discovery of an evolutionarily ancient form of cell death, ferroptosis, through a pharmacological approach in 2012, both the molecular mechanisms of ferroptosis and potential application studies have been popular research topics. Although the upstream 


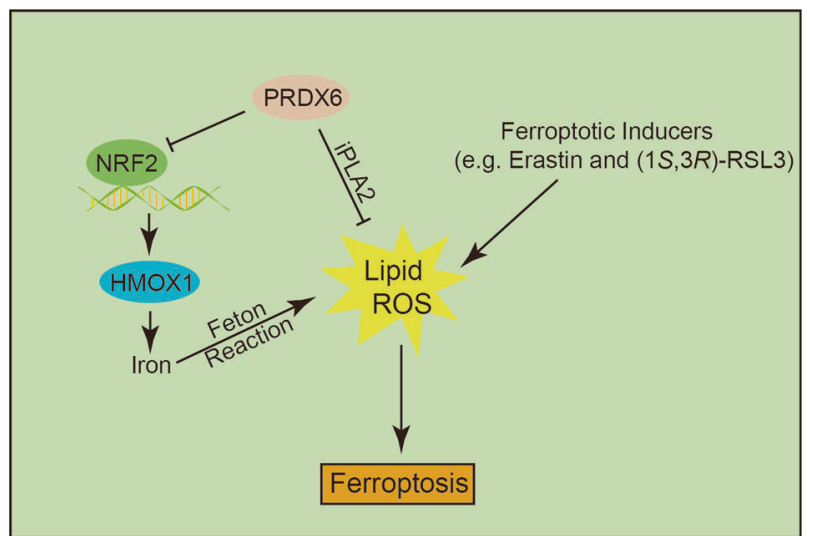

Fig. 5 The schematic representation of PRDX6 negatively regulating ferroptosis working model

mechanism of ferroptosis has been well-characterized, the mechanism of how cells die during ferroptosis remains unknown [6]. For example, how does $\mathrm{LOOH}$ lead to ferroptosis? Regarding this question, it is important to identify other regulators and pathways for removing lipid ROS.

Given that PRDX6 is able to complete the regeneration of peroxidized cell membranes following an oxidative event, our present study further expands the potential role of PRDX6 in ferroptotic oxidative stress. Compared with other peroxidases of the peroxiredoxin family, PRDX6 has a unique form of enzymatic activity as a $\mathrm{Ca}^{2+}$-independent phospholipase $\mathrm{A} 2$ [32]. It is able to catalyze the hydrolysis of the oxidized sn-2 PUFAs in PL hydroperoxide. In our study, Erastin-induced $\mathrm{LOOH}$ was further enhanced when PRDX6-PLA2 activity was inhibited by MJ33. In other words, PRDX6 has the ability to remove $\mathrm{LOOH}$ during the ferroptotic process. While we demonstrated that MJ33 could promote Erastin-induced $\mathrm{LOOH}$, this effect was not as obvious as PRDX6 silencing did. In addition, the phospholipase A2 activity of PRDX6 only partially contributes to the repair of peroxidized cell membranes. Thus, our present results could not exclude an important role of PL hydroperoxide GPx activity and LPC acyl transferase activity in the ferroptotic process. Previous studies have indicated that PRDX6-deficient mice develop normally but are susceptible to oxidative stress [33]. Therefore, it is reasonable and useful to investigate the accurate role of different PRDX6enzymatic activities during the ferroptotic process through ectopic expression of wild type or inactive mutant PRDX6 in PRDX6 $^{-1-}$ mouse embryonic fibroblast cells (PRDX6 ${ }^{-1-}$ MEFs).

Since its discovery, ferroptosis has been shown not only to be an attractive anticancer mechanism but also to be involved in a variety of pathophysiological settings. For example, another ferroptotic inhibitor, liproxstatin-1, is able to mitigate tissue injury in ischemia/reperfusion-induced liver injury [14]. Moreover, ferrostatin-1 prevented cell death in an ex vivo model of rhabdomyolysis-induced acute kidney injury [34]. These results suggest that ferroptosis is implicated in these pathological conditions. In addition, it has been reported that PRDX6 overexpression attenuates LPS-induced acute kidney injury in vivo [35]. Based on these facts, it is important to further investigate the relationship between negative regulatory effects of PRDX6 on ferroptosis and its role in ameliorating some pathological diseases.

In addition, we observed that PRDX6 silencing promotes NRF2 expression and activation in ferroptosis. In recent years, different groups have discovered contradictory roles of heme oxygenase-1 in ferroptosis. Dr. Tang's laboratory [17] suggested that NRF2 protects hepatocellular carcinoma cells against ferroptosis partially through the activated transcription of heme oxygenase-1, while another laboratory [30] showed that HMOX1 enhanced Erastin- induced ferroptosis through metabolites of heme (i.e., hemin and CO-releasing molecules) in HT-1080 fibrosarcoma cells. Although the reason for explaining the opposite effect of NRF2 and HMOX1 on ferroptosis is far from determined, our results interestingly indicate that $H M O X 1$ is the only gene that was significantly unregulated among six classic NRF2 target genes involved in heme and iron metabolism and GSH synthesis, suggesting that HMOX1 could be the actual downstream link in response to PRDX6-silencing-enhanced ferroptosis. Moreover, our data may indicate an exciting possibility that the selectivity of NRF2 downstream gene expression is governed by other factors, which should be further discovered.

In conclusion, we report that PRDX6 is an essential negative regulator of sensitivity to ferroptosis. The knockdown of PRDX6 significantly enhances ferroptosis inducer-triggered $\mathrm{LOOH}$ and ferroptotic cell death in various cancer cell lines, while overexpression of PRDX6 inhibits Erastin-triggered LOOH and ferroptotic cell death. Mechanistically, PRDX6 not only removes a portion of induced $\mathrm{LOOH}$ through its phospholipase $\mathrm{A} 2$ activity but also suppresses HMOX1 function, resulting in decreased iron levels and subsequent Fenton chemistry (Fig. 5). Future experiments on the importance of other PRDX6 enzymatic activities in ferroptosis will lead to an improved understanding of PRDX6 in ferroptosis. In addition, our discovery has provided new insights into possible combinational cancer therapy targeting both PRDX6 and ferroptosis.

\section{ACKNOWLEDGEMENTS}

This work was supported by grants from the National Natural Science Foundation of China (No. 81872885 to Ji Cao; No. 81625024 to Bo Yang), the Department of Education of Zhejiang Province (Y201430401 to Ji Cao), the Talent Project of Zhejiang Association for Science and Technology (No. 2018YCGC002 to Ji Cao) and the Project of Zhejiang Medical Science and Technology (2015KYA141 to Yu-cai Hong). We thank Dr. Nicole Spiegelman and Dr. Zhen Tong for language editing.

\section{AUTHOR CONTRIBUTIONS}

JC, MDY, BY, QJH, and BL designed the research project; $B L$ and $\mathrm{XBC}$ performed the experiments; JC and $\mathrm{BL}$ analyzed the data; $\mathrm{HZ}$ and $\mathrm{YCH}$ contributed the reagents; JC, $\mathrm{BL}$, and $\mathrm{XBC}$ wrote the paper.

\section{ADDITIONAL INFORMATION}

The online version of this article (https://doi.org/10.1038/s41401-019-0233-9) contains supplementary material, which is available to authorized users.

Competing interests: The authors declare no competing interests.

\section{REFERENCES}

1. Dixon SJ, Lemberg KM, Lamprecht MR, Skouta R, Zaitsev EM, Gleason CE, et al. Ferroptosis: an iron-dependent form of nonapoptotic cell death. Cell. 2012;149:1060-72.

2. Yang WS, Kim KJ, Gaschler MM, Patel M, Shchepinov MS, Stockwell BR. Peroxidation of polyunsaturated fatty acids by lipoxygenases drives ferroptosis. Proc Natl Acad Sci USA. 2016;113:E4966-75.

3. Doll S, Proneth B, Tyurina YY, Panzilius E, Kobayashi S, Ingold I, et al. ACSL4 dictates ferroptosis sensitivity by shaping cellular lipid composition. Nat Chem Biol. 2017;13:91-8.

4. Kagan VE, Mao G, Qu F, Angeli JP, Doll S, Croix CS, et al. Oxidized arachidonic and adrenic PEs navigate cells to ferroptosis. Nat Chem Biol. 2017;13:81-90.

5. Stockwell BR, Friedmann Angeli JP, Bayir H, Bush Al, Conrad M, Dixon SJ, et al. Ferroptosis: a regulated cell death nexus linking metabolism, redox biology, and disease. Cell. 2017;171:273-85.

6. Lu B, Chen XB, Ying MD, He QJ, Cao J, Yang B. The role of ferroptosis in cancer development and treatment response. Front Pharmacol. 2017;8:992.

7. Yang WS, Stockwell BR. Ferroptosis: death by lipid peroxidation. Trends Cell Biol. 2016;26:165-76.

8. Xie $Y$, Hou W, Song $X, Y u$ Y, Huang J, Sun $X$, et al. Ferroptosis: process and function. Cell Death Differ. 2016;23:369-79. 
9. Jiang L, Kon N, Li T, Wang SJ, Su T, Hibshoosh H, et al. Ferroptosis as a p53mediated activity during tumour suppression. Nature. 2015;520:57-62.

10. Xie $Y$, Zhu S, Song $X$, Sun X, Fan Y, Liu J, et al. The tumor suppressor $p 53$ limits ferroptosis by blocking DPP4 activity. Cell Rep. 2017;20:1692-704.

11. Yang WS, SriRamaratnam R, Welsch ME, Shimada K, Skouta R, Viswanathan VS, et al. Regulation of ferroptotic cancer cell death by GPX4. Cell. 2014;156: 317-31.

12. Roh JL, Kim EH, Jang HJ, Park JY, Shin D. Induction of ferroptotic cell death for overcoming cisplatin resistance of head and neck cancer. Cancer Lett. 2016;381:96-103.

13. Yu Y, Xie Y, Cao L, Yang L, Yang M, Lotze MT, et al. The ferroptosis inducer Erastin enhances sensitivity of acute myeloid leukemia cells to chemotherapeutic agents. Mol Cell Oncol. 2015;2:e1054549.

14. Friedmann Angeli JP, Schneider M, Proneth B, Tyurina YY, Tyurin VA, Hammond VJ, et al. Inactivation of the ferroptosis regulator $\mathrm{Gpx} 4$ triggers acute renal failure in mice. Nat Cell Biol. 2014;16:1180-91.

15. Dixon SJ, Patel DN, Welsch M, Skouta R, Lee ED, Hayano M, et al. Pharmacological inhibition of cystine-glutamate exchange induces endoplasmic reticulum stress and ferroptosis. eLife. 2014;3:e02523.

16. Sun $X$, Ou Z, Xie M, Kang R, Fan Y, Niu X, et al. HSPB1 as a novel regulator of ferroptotic cancer cell death. Oncogene. 2015;34:5617-25.

17. Sun X, Ou Z, Chen R, Niu X, Chen D, Kang R, et al. Activation of the p62-Keap1NRF2 pathway protects against ferroptosis in hepatocellular carcinoma cells. Hepatology. 2016;63:173-84.

18. Dixon SJ, Winter GE, Musavi LS, Lee ED, Snijder B, Rebsamen M, et al. Human haploid cell genetics reveals roles for lipid metabolism genes in nonapoptotic cell death. ACS Chem Biol. 2015;10:1604-9.

19. Rhee SG, Chae HZ, Kim K. Peroxiredoxins: a historical overview and speculative preview of novel mechanisms and emerging concepts in cell signaling. Free Radic Biol Med. 2005;38:1543-52.

20. Rhee SG, Kang SW, Chang TS, Jeong W, Kim K. Peroxiredoxin, a novel family of peroxidases. IUBMB Life. 2001;52:35-41.

21. Fisher AB, Vasquez-Medina JP, Dodia C, Sorokina EM, Tao JQ, Feinstein SI. Peroxiredoxin 6 phospholipid hydroperoxidase activity in the repair of peroxidized cell membranes. Redox Biol. 2018;14:41-6.

22. Fisher $A B$. Peroxiredoxin 6 in the repair of peroxidized cell membranes and cell signaling. Arch Biochem Biophys. 2017;617:68-83.
23. Fisher AB, Dodia C, Sorokina EM, Li H, Zhou S, Raabe T, et al. A novel lysophosphatidylcholine acyl transferase activity is expressed by peroxiredoxin 6. J Lipid Res. 2016;57:587-96.

24. Xu Z, Jin Y, Yan H, Gao Z, Xu B, Yang B, et al. High-mobility group box 1 proteinmediated necroptosis contributes to dasatinib-induced cardiotoxicity. Toxicol Lett. 2018;296:39-47.

25. Dolma S, Lessnick SL, Hahn WC, Stockwell BR. Identification of genotype-selective antitumor agents using synthetic lethal chemical screening in engineered human tumor cells. Cancer Cell. 2003;3:285-96.

26. Yang WS, Stockwell BR. Synthetic lethal screening identifies compounds activating iron-dependent, nonapoptotic cell death in oncogenic-RAS-harboring cancer cells. Chem Biol. 2008;15:234-45.

27. Zilka O, Shah $R$, Li B, Friedmann Angeli JP, Griesser $M$, Conrad $M$, et al. On the mechanism of cytoprotection by ferrostatin-1 and liproxstatin-1 and the role of lipid peroxidation in ferroptotic cell death. ACS Cent Sci. 2017;3:232-43.

28. Chowdhury I, Mo Y, Gao L, Kazi A, Fisher AB, Feinstein SI. Oxidant stress stimulates expression of the human peroxiredoxin 6 gene by a transcriptional mechanism involving an antioxidant response element. Free Radic Biol Med. 2009;46:146-53.

29. Kansanen E, Kuosmanen SM, Leinonen H, Levonen AL. The Keap1-Nrf2 pathway: mechanisms of activation and dysregulation in cancer. Redox Biol. 2013;1:45-9.

30. Kwon MY, Park E, Lee SJ, Chung SW. Heme oxygenase-1 accelerates Erastininduced ferroptotic cell death. Oncotarget. 2015;6:24393-403.

31. Lanceta L1, Li C, Choi AM, Eaton JW. Haem oxygenase-1 overexpression alters intracellular iron distribution. Biochem J. 2013;449:189-94.

32. Chen JW, Dodia C, Feinstein SI, Jain MK, Fisher AB. 1-Cys peroxiredoxin, a bifunctional enzyme with glutathione peroxidase and phospholipase $A 2$ activities. J Biol Chem. 2000;275:28421-7.

33. Fatma N, Kubo E, Sharma P, Beier DR, Singh DP. Impaired homeostasis and phenotypic abnormalities in Prdx6-/-mice lens epithelial cells by reactive oxygen species: increased expression and activation of TGFbeta. Cell Death Differ. 2005:12:734-50.

34. Skouta R, Dixon SJ, Wang J, Dunn DE, Orman M, Shimada K, et al. Ferrostatins inhibit oxidative lipid damage and cell death in diverse disease models. J Am Chem Soc. 2014;136:4551-6.

35. Lee DH, Park JH, Han SB, Yoon DY, Jung YY, Hong JT. Peroxiredoxin 6 overexpression attenuates lipopolysaccharide-induced acute kidney injury. Oncotarget. 2017;8:51096-107. 